\title{
BMJ Open Risk factors associated with quad bike crashes: a protocol for systematic review of observational studies
}

\author{
Preetha Menon (D) , ${ }^{1}$ Mohamed El-Sadig (D) , ${ }^{1}$ Moien AB Khan (D) , ${ }^{2}$ \\ Linda Östlundh (D) , ${ }^{3}$ Marwan El-Deyarbi, ${ }^{4}$ Rami H Al-Rifai (D) , ${ }^{1}$ Michal Grivna (D) ${ }^{1}$
}

To cite: Menon P, El-Sadig M, AB Khan M, et al. Risk factors associated with quad bike crashes: a protocol for systematic review of observational studies. BMJ Open 2021;11:e044456. doi:10.1136/ bmjopen-2020-044456

- Prepublication history and additional material for this paper are available online. To view these files, please visit the journal online (http://dx.doi. org/10.1136/bmjopen-2020044456).

Received 03 September 2020 Revised 16 February 2021 Accepted 01 March 2021

\section{Check for updates}

(c) Author(s) (or their employer(s)) 2021. Re-use permitted under CC BY-NC. No commercial re-use. See rights and permissions. Published by BMJ.

${ }^{1}$ Institute of Public Health, United Arab Emirates University, College of Medicine and Health Sciences, Al Ain, UAE

${ }^{2}$ Department of Family Medicine, UAE University College of Medicine and Health Sciences, Al Ain, Abu Dhabi, UAE ${ }^{3}$ National Medical Library, United Arab Emirates University College of Medicine and Health Sciences, Al Ain, Abu Dhabi, UAE ${ }^{4}$ Department of Pharmacology, United Arab Emirates University College of Medicine and Health Sciences, Al Ain, UAE

Correspondence to Dr Mohamed El-Sadig; msadig@uaeu.ac.ae

\section{ABSTRACT}

Introduction Quad bikes are four-wheeled vehicles, driven off-road on uneven terrains by farmers for work or young adults for leisure. Quad bike accidental crashes result mostly due to the unique ecosystem of uneven terrain, where these unstable vehicles are commonly driven, in addition to numerous distinctive sociodemographic characteristics related to drivers. This is a protocol for a systematic review of observational studies from all geographical regions and demographic groups in the world to summarise the common risk factors relating to quad bike crashes.

Methods and analysis A comprehensive search for the literature on quad bike crashes and related injuries will be conducted in six electronic databases: PubMed, Embase, Scopus, Web of Science, IEEE and PsycINFO. Proquest Dissertation and Thesis, OpenGrey and BASE will be searched for grey literature. Five researchers will be involved in the screening, and the review of full text articles, using the inclusion and exclusion criteria. Disagreements between reviewers will be resolved by discourse. Three researchers will help resolving conflicts that may arise during the screening process and will resolve eventual conflicts identified in the process with the help of the systematic review software 'Covidence' for automatic deduplication and blinded screening. Information on crashes leading to injuries and death, target population characteristics and risk factors involved will be extracted from eligible articles in addition to the assessment of the quality of the researched articles. Ethics and dissemination Since this is a systematic review of published literature, a formal ethical approval is not needed. Results of the review will be disseminated through peer-reviewed publications, conference presentations and reports to the concerned authorities. PROSPERO registration number CRD42020170245.

\section{BACKGROUND}

Quad bikes are four-wheeled, off-road vehicles, characterised by low air pressure tyres, saddle seat and handlebars. They are distinct in their narrow wheel base and have been widely used for farm work and more recently, for recreational purposes. ${ }^{1}$ Regular quad bike use is associated with increased aerobic fitness, improved blood lipid profile and lower risk of metabolic syndrome. ${ }^{2}$ In terms of

\section{Strengths and limitations of this study}

- The study will follow the Preferred Reporting Items for Systematic Reviews and Meta-Analyses Protocols (PRISMA) guidelines.

- The study will present a transparent and rigorous screening and review methodology involving multiple independent researchers, using the Covidence software (Veritas Health Innovation, 2020).

- The study will help to fill up the evidence gap, using the public health approaches to identify the risk factors for quad bike crashes and injuries, namely the Haddon Matrix and the Ecological Model.

- Limitations may include the low level of evidence generated from the observational studies.

health benefits, quad bikes can be compared with other outdoor sports like bicycling. However, in terms of crash risk and severity of the resulting injuries associated with quad bikes, it is said to be comparable to motor vehicle injuries. ${ }^{3}$

Quad bike crashes constitute a growing public health problem at the individual and societal levels, due to their adverse debilitating health consequences, including disability, injury and death, in addition to the direct and indirect costs related to medical treatment and work-related losses from disability and absenteeism. ${ }^{34}$ As such, the prevention of quad bike injuries is important in order to preserve and maintain the health and wellbeing of the society and to reduce the unnecessary costs of these needless injuries.

Quad bikes have an inherent instability in their design. When used in desert and rugged terrain, they are characterised by high uncertainty in the driving surface. The unpaved terrain, narrow wheel base, high centre of gravity and large engine size makes them prone to rollover and collision, even at low speed. ${ }^{35}$ The magnitude of risk while riding a quad bike is exacerbated in the context of outdoor sport and the demographic group involved. ${ }^{6}$ Young 
adults, adolescents and children are known to carry an additional psychological impulse for risk taking, high speed and aggressive driving. ${ }^{57}$ Thus, the risk factors associated with quad bikes are more related to the environment where they are driven, the unique vehicular engineering and the driver characteristics. It is therefore obvious that the conventional risk factors associated with motor vehicle injuries cannot be blindly extrapolated to quad bike injuries.

Any safety intervention to control unintentional injuries begins with a robust risk assessment study, which requires an understanding of the primary risk factors associated and involved with the activity, leading to injuries. ${ }^{8}$ Therefore, a systematic and an objective review of the existing scientific literature is imperative to highlight and identify the possible risk factors associated with quad bike crashes and injuries. Unlike road traffic injuries and occupational injuries, however, there has been limited research on the risk factors of quad bike injuries. A comprehensive search for systematic reviews on quad bike injuries, by the authors, resulted in only one review, which have been carried out in $2018 .{ }^{9}$ However, the review focused only on helmet use among quad bike users and did not provide an exhaustive list of other risk factors.

In the absence of a standardised methodology for systematic review of the risk factors of quad bikes crashes and injuries, it was a challenge to develop a protocol to meet the objectives of the study and maintaining the quality of the review. ${ }^{10}$ This study protocol will attempt to develop a more rigorous method to review studies on quad bike crashes and injuries, using the predominant observational studies.

The results from this review will help to understand the risk factors responsible for quad bike crashes and injuries, especially, the factors that can be reduced, controlled or eliminated altogether, using interventional safety programmes.

\section{Research question}

As the review aims to identify and classify the risk factors for crashes due to quad bike use, the research question follows the Population-Exposure-Control-Outcome format. ${ }^{11}$ The target population include people of any region, age, gender or occupation, who use quad bikes on paved roads and uneven terrain. The exposure under focus involves driving a quad bike or similar four-wheeled saddle-seated, on paved roads or uneven terrain. The control would be those who are not driving quad bikes or those who have not crashed. The health outcome of interest would be any quad bike crashes that could lead to injuries, disability or death. Thus, the research question that drives this review would be: What are the risk factors for crashes, associated with quad bike use on paved roads and uneven terrain, leading to injury, disability and death among quad bike drivers?

\section{OBJECTIVES}

1. To identify the risk factors and protective factors of crashes related to quad bike use on paved roads and uneven terrain.

2. To classify the risk factors into personal, engineering and environmental risk factors.

3. To analyse these risk factors using the Haddon Matrix and the ecological model to determine the primary, secondary and tertiary interventional measures needed to control the problem.

\section{METHODS}

This protocol will follow the Preferred Reporting Items for Systematic Reviews and Meta-Analyses Protocols (PRISMA-P) 2015 guidelines. ${ }^{12}$

\section{Inclusion criteria}

Study design

The review will comprise of all observational studies, including cross-sectional analytical studies, case-control studies, surveys, mixed methods and qualitative studies. Policy documents, especially those describing legalregulatory environment of the region will be included to identify risk factors related to social and policy environment.

Risk factors refer to factors that either predispose or protect the quad bike driver from crash. Both predisposing and protective factors will be included in the review with no preference given to either of the two categories. All effect measures quantifying and identifying risk factors such as relative risk, Odds Ratio (OR), risk difference towards the main outcome will be included in the review.

\section{Study population}

All quad bike riders who experienced crashes are subjects for the review. Therefore, studies and reports targeting quad bike drivers of all regions, age groups, occupational groups and gender will be included in the review. The vehicles under review are open, saddle seated four wheeled off-terrain vehicles. They may or may not have rollover protection structures like roll cages, quad bars or lifeguards. The comparison group would be those who do not drive quad bikes or who have not experienced crash from quad bike use.

\section{Study outcome}

The health effects of crashes will not be specified as they range from injury, disability to death within 48 hours of the event. The focus of the review will be on the risk factors associated with quad bike crashes, and not any specific health effect or outcome. All studies and reports related to quad bike crashes resulting in outcomes ranging from no injury to disability or death will be included in the review. 


\section{Study environment}

The results of this review will be systematically evaluated, thematically synthesised and analysed using the Haddon Matrix which helps to identify the risk factors related to personal attributes, vector or agent attributes and environmental attributes; before, during and after injury or death ${ }^{13}$. We define uneven terrain as those without smooth surface or have surface irregularities. ${ }^{14}$ These include slopes, sand dunes, dirt track, unpaved surfaces, gravel, grass or agricultural workplace environment. The vehicle/vector-related risk factors of a quad bike on paved roads are clearly different from those on unpaved roads. ${ }^{15} 16$ The vehicle-related protective features for on-road safety, like seatbelts, sometimes contradicts basic quad bike protective features on uneven terrain like active dismount. ${ }^{17}$ This is because the injury mechanism on roads, due to head on collision crashes, are different from that of off-road injuries (rollover, ejection). ${ }^{18} 19$ Therefore, to increase the specificity of the review, articles describing quad bikes crashes on paved roads and uneven terrain will be included but synthesised separately.

\section{Exclusion criteria}

\section{Source}

Blog posts, opinion articles, editorials, advertisement brochure and news articles that do not cite primary source or research findings will be excluded from data extraction as they may compromise the data quality. However, they will be included to the screening list as handsearched references.

\section{Outcome}

Articles related to the mechanics of the vehicle but not related to crash risk factors, and those related to armed combat, without reference to crash or stability, will be excluded from this review. Studies focusing on long term exposure and chronic health outcomes as well as those on positive health outcomes will be excluded from this study.

\section{Risk environment}

Articles related to other off-road vehicles like dirt bikes and snowmobiles will be excluded as they present heterogeneous vehicular and terrain-related risk factors.

\section{Search strategy}

\section{Developing research question and search query domains}

The research question for the review was developed using the population-exposure-outcome parameters sought to be appropriate for the etiologic review methodology. The research question was divided into functional areas of population involved and exposure factors.

Our focus will be on increasing the sensitivity of the search by identifying studies on quad bike users of any region, demographic group, occupation group and gender. Thus, the search query will not include any specific population-related terms associated with region, gender, occupation group or age groups. This was tested with presearches that showed no improvement in the sensitivity of the search when including population-related keywords.
Crash risk was defined as any personal, vehicle engineering or environmental factors that could directly or indirectly influence the occurrence of quad bike crashes, resulting from driving quad bikes on paved roads or uneven terrain. Search terms used will include injury related causality or risk inclusive of all risk levels. It will also include terms for protective risk factors that have been reported in the published research to reduce the crash risk.

Thus, our search terms will focus on the nomenclature related to quad bikes to specify the target population and broad risk related terms. Our search strings will include vehicle related terms such as "Quad bikes", "All terrain Vehicle", "Four wheel ATV" etc., and risk related terms such as "Safety", "Risk" or "Cause". Detailed search string is available in online supplemental file. All selected search terms will be searched in a combination of fields: title, abstract and MeSH (Pubmed Medical Subject Headings vocabulary thesaurus for indexing articles) when available for the best possible information retrieval. No filters or limitations will be used for the search. Handscreening of the reference list of studies that have been included will also be conducted. This combination of target population and risk exposure keywords yielded maximum number of articles on presearch trials. Detailed search string is available in online supplemental file.

The published literature will be the primary sources of our research through a systematic search in PubMed (The US National library of Medicine), Scopus, Embase, PsycINFO, IEEE (Institute of Electrical \& Electronic Engineers) and Web of Science. The grey literature will be sourced from Proquest Thesis and Dissertations, OpenGrey and BASE (Bielefeld Academic Search Engine). A search log with detailed search strategies, results and notes for each database included will be appended to the review. Pre-searches in PubMed to identify search terms and develop the search string was performed in MayJune 2020 and the full search will be completed in March 2021. A search update of all information sources will be performed ahead of the manuscript submission to ensure that all recent studies are covered in the review. An up-todate copy of the pre-searches in PubMed is available in the online supplemental materials.

We will not apply exclusion criteria based on the date of publication, place of publication or language of publication. Articles in all languages will be included and translated into the English language during the review to identify risk factors. The literature on quadbikes, in languages other than the English langauge, will be searched and screened using available online translation tools such as "GoogleTranslate" in addition to the language skills of the coauthors. When a paper meet the inclusion criteria, a qualified translator with the relevant language skill, will be approached for the full translation of the article. 


\section{Data extraction and management}

\section{Screening and study selection}

All studies identified in the databases search will be exported to the systematic review software Covidence (Veritas Health Innovation, 2020) for automatic deduplication and blinded screening.

Two reviewers will screen the titles and abstracts against the inclusion and exclusion criteria with the help of blind screening functions in Covidence (Veritas Health Innovation, 2020). Any conflict identified by the software will be resolved by a third reviewer. Full text PDF files of the papers selected for further full-text screening will be uploaded to Covidence by the National Medical Library staff at the UAE University.

Similarly, full-text review and study selection will be conducted by two independent reviewers with a third external reviewer for conflict resolution. Studies included in the full text screening will be documented with the reason for exclusion in a PRISMA flow diagram.

This review will screen reports, white papers, thesis, editorials and policy briefs, to identify primary research publications that might have been missed from the databases.

\section{Data extraction}

The summary of variables in which data will be extracted are: author, journal, year of publication, study setting, study design, geographical location, studied population or demographic group, vehicle specification, health outcome, risk factors, safety protective factors, risk factor characteristics, strength of association and safety intervention methods. Data extraction will be carried out by two reviewers using data extraction form developed in Covidence. Pilot data extraction for the 25 most cited articles will be followed by evaluation of the data extraction tool and correction by the team. Five reviewers will assess quality of study, and will check for transcription errors, incomplete data and classification errors. Every article will be reviewed to minimise errors and identify bias.

\section{Risk of bias in primary study}

Appropriate population acknowledged biases and addressed biases would be the basic criteria to assess the validity of the study results. Precision of measurement instrument, statistical analysis and sound sampling method would be an added bonus, but not necessarily a prerequisite for these studies. ${ }^{20}$

This review is expected to identify a wide variety of study designs like qualitative studies, preinterventionspostinterventions, non-randomised interventions and cross-sectional analytical studies. One validated tool to assess all these study methodologies is the Mixed Method Appraisal Tool. ${ }^{21}$ Five reviewers will assess the quality of the data employing validated Mixed Method Appraisal Tool to check their eligibility for data extraction.
Data synthesis

All selected studies will be included in the synthesis. Data extracted from translated articles will be validated by language experts. Analysis will follow the narrative meta-summary approach and data synthesis will involve compiling and classifying risk factors according to the Haddon Matrix and ecological model. This review will use the Haddon Matrix framework to classify the risk factors related to quad bike crashes and injuries. Developed by William Haddon in $1970,{ }^{22}{ }^{23}$ the matrix looks at risk factors related to personal attributes, vector attributes and environmental attributes, in relation to the time of the incident occurrence; before, during and after the incident (whether an injury or death). The combination between the epidemiological triad and levels of prevention, gives the analyst a way to look at planning for injury prevention and intervention strategies by phases in time of the event. As such, the framework help to identify the risk factors that can be controlled before, during and after the event, through educational, engineering and environmental interventional measures. ${ }^{24}$

Data compilation will be based on the person, vehicle engineering and environment risk factors and health outcomes that might emerge from the review data. Studies will be classified according to the quality of data and could influence data synthesis. The results will be presented in a non-quantitative narrative overview.

\section{Patient and public involvement}

No patients or individuals from the public will be involved in this review. Patient consent is not required for this review.

\section{Ethics and dissemination}

Since this research is confined only to the published literature and does not involve any direct contact with human subjects, it will not need an ethics clearance by the university human research ethics committee. Yet, the research will follow the ethics guidelines for systematic review described by Wagner and Wiffen. ${ }^{25}$ The review will include studies of all regions, sociodemographic groups and occupations. It will not exclude studies based on their results or due to conflict with other study results. In the absence of meta-analysis, only significant results will be extracted and presented. However, if any conflicting results from multiple studies were cited, they will be presented without further filtering.

The study will use the software Covidence (Veritas Health Innovation, 2020) for the screening and extraction of data to ensure the blinding and full transparency of the screening and selection process. The review will identify redundant publications with the same results. The scientific status of the final selected articles published in Open Access journals will be analysed with the help of Predatory reports from Cabells Scholarly Analytics. ${ }^{26}$

Since the review aims to inform policy makers in designing interventions, the extracted data will need to be synthesised and presented, using the conceptual 
framework of the Haddon Matrix to identify the risk factors related to personal attributes, vector or agent and environmental attributes; before, during and after injury or death. The results of the study will be published in peer-reviewed journals. The results from the review will also be presented in workshops and meetings with relevant stakeholders and policy-makers. Information will be presented in simple tables and infographics.

\section{OUTPUT}

The study will present the PRISMA flow diagram, summarising the search and study selection process. A comprehensive table of study characteristics will be given along with study quality rating. It will summarise the risk factors identified in each study. All information will be synthesised to classify and evaluate the relative importance of the different risk factors, using the Haddon Matrix and the Haddon's strategies for prevention. 22232728

\section{BIAS MINIMISATION}

Multiple databases will be searched to get published literature as well as grey literature. Publication bias is minimised by a broad inclusion criteria of study design, type of publication and adverse health outcomes. Publication bias is further reduced in the absence of treatment effect or meta-analysis, where results of all sources and design are given equal weightage. Selection bias will be assessed by quality checks on a sample of included articles and excluded articles assessing uniform adoption of inclusion and exclusion criteria. Data extraction process will be audited by five reviewers MES, MABK, MG, RHA-R and LÖ to ensure adherence to inclusion and exclusion criteria, minimise transcription errors and bias. All information on study details and extraction process will be shared in the public domain during publication. Information bias on the validity of study results will be assessed by extracting information on the objective measurement tool and statistical analysis accounting for confounders. In addition to the source, the screening process by multiple reviewers will be blinded using the systematic review software Covidence.

\section{DISCUSSION AND POSSIBLE CHALLENGE}

It is expected that most of the studies which will be reviewed would be predominantly descriptive studies, describing a well-defined population, with well-defined exposure and a well- defined crash outcome. The population will comprise all drivers of four-wheeled, all-terrain quad bikes. The outcomes would include crashes leading to accidental injuries, disability or death, while driving quad bikes on paved roads or uneven terrain. Though the wide range of outcomes might identify a common set of injury risk factors, they might still not allow metaanalysis for risk association. However, all modifiable risk factors that increase the risk of these health effects will be recorded and synthesised in this review. This systematic review protocol does not meet the Populatin Intervention Comparison Outcome (PICO) framework for formulating research questions and, therefore, would need a different approach appropriate for aetiological studies. The protocol was designed according to PRISMA-P guidelines with influence of ROBIS and Amstar assessment tools. ${ }^{12}{ }^{20}{ }^{29}$ In the absence of a standardised risk of bias tool for systematic review of observational studies and any competent scale for the assessment of qualitative studies, we will develop an appropriate method for our research objective. Notwithstanding, the specific populations involved in quad bike entertainment and the resulting health effects, the search for risk factors could be very extensive. However, the information extracted from these articles, using objective measures, could help to elucidate the risk factors of quad bike crashes and the mitigation strategies necessary to reduce their adverse health effects. Despite the methodological weaknesses of using observational data to synthesise evidence, a rigorous systematic review could help to provide new opportunities for quad bike injury prevention research. Additionally, the research may also provide new research evidence to policy-makers for interventional safety strategies.

Contributors PM, MES, MABK, RHA-R, MG and LÖ were involved in conceptualisation and protocol development. PM, LO and MES were involved in developing search strategy and grey literature search. Literature search will be conducted by PM and LÖ. PM, MABK, MED will be involved in screening. MABK, MED, RHA-R, MG and MES will resolve conflicts. PM and MED will extract data, which will be validated by MES, MABK, MG, RHA-R and LÖ.

Funding This work is supported by (College of Medicine \& Health Sciences at UAE University Internal Grants - 2019).

Competing interests None declared.

Patient consent for publication Not required.

Provenance and peer review Not commissioned; externally peer reviewed.

Supplemental material This content has been supplied by the author(s). It has not been vetted by BMJ Publishing Group Limited (BMJ) and may not have been peer-reviewed. Any opinions or recommendations discussed are solely those of the author(s) and are not endorsed by BMJ. BMJ disclaims all liability and responsibility arising from any reliance placed on the content. Where the content includes any translated material, BMJ does not warrant the accuracy and reliability of the translations (including but not limited to local regulations, clinical guidelines, terminology, drug names and drug dosages), and is not responsible for any error and/or omissions arising from translation and adaptation or otherwise.

Open access This is an open access article distributed in accordance with the Creative Commons Attribution Non Commercial (CC BY-NC 4.0) license, which permits others to distribute, remix, adapt, build upon this work non-commercially, and license their derivative works on different terms, provided the original work is properly cited, appropriate credit is given, any changes made indicated, and the use is non-commercial. See: http://creativecommons.org/licenses/by-nc/4.0/.

\section{ORCID iDs}

Preetha Menon http://orcid.org/0000-0001-5999-1249

Mohamed El-Sadig http://orcid.org/0000-0001-5795-8189

Moien AB Khan http://orcid.org/0000-0003-4970-4618

Linda Östlundh http://orcid.org/0000-0001-5091-604X

Rami H Al-Rifai http://orcid.org/0000-0001-6102-0353

Michal Grivna http://orcid.org/0000-0003-2349-0922 


\section{REFERENCES}

1 Clay L, Hay-Smith J, Treharne G, et al. "There are risks to be taken and some just push it too far": how farmers perceive quad-bike incident risk. Aust N Z J Public Health 2016;40:55-61.

2 Burr JF, Jamnik VK, Gledhill N. Physiological fitness and health adaptations from purposeful training using off-road vehicles. Eur $J$ Appl Physiol 2011;111:1841-50.

3 Bohl S. All- Terrain Vehicle Related Injuries and Hospitalization : An Examination of the Influence of Age and Substance Abuse [Doctoral Dissertation]. Minnesota: Walden University, 2010.

4 Lower T, Pollock K, Herde E. Australian quad bike fatalities: what is the economic cost? Aust N Z J Public Health 2013;37:173-8.

5 Milosavljevic S, McBride DI, Bagheri N, et al. Factors associated with quad bike loss of control events in agriculture. Int $J$ Ind Ergon 2011;41:317-21.

6 Vanlaar W, McAteer H, Brown S, et al. Injuries related to off-road vehicles in Canada. Accid Anal Prev 2015;75:264-71.

7 Khan MAB, Grivna M, Nauman J, et al. Global incidence and mortality patterns of pedestrian road traffic injuries by sociodemographic index, with forecasting: findings from the global burden of diseases, injuries, and risk factors 2017 study. Int J Environ Res Public Health 2020;17:2135.

8 Siraj NB, Fayek AR. Risk identification and common risks in construction: literature review and content analysis. J. Constr. Eng. Manage. 2019;145:03119004

9 Rattan R, Joseph D'Andrea K, Dente CJ, et al. Prevention of all-terrain vehicle injuries: a systematic review from the eastern association for the surgery of trauma. J Trauma Acute Care Surg 2018;84:1017-26.

10 JBI. JBI Reviewer's Manual: Introduction to etiological evidence and systematic reviews, 2020. Available: https://reviewersmanual. joannabriggs.org/

11 Morgan RL, Whaley P, Thayer KA, et al. Identifying the PECO: a framework for Formulating good questions to explore the association of environmental and other exposures with health outcomes. Environ Int 2018;121:1027-31.

12 Moher D, Shamseer L, Clarke M, et al. Preferred reporting items for systematic review and meta-analysis protocols (PRISMA-P) 2015 statement. Syst Rev 2015;4:1-9.

13 World Health Organization. World report on road traffic injury prevention. Geneva: World Health Organization, The World Bank, 2004. ISBN: 9241562609.
14 Hicks D, Grzebieta R, Mongiardini M, et al. Investigation of when quad bikes rollover in the farming environment. Saf Sci 2018;106:28-34.

15 Lord S, Tator CH, Wells S. Examining Ontario deaths due to all-terrain vehicles, and targets for prevention. Can J Neurol Sci 2010;37:343-9.

16 Lagerstrom E, Magzamen S, Stallones L, et al. Understanding risk factor patterns in ATV fatalities: a recursive partitioning approach. $J$ Safety Res 2016;59:23-31.

17 Khorsandi F, Ayers PD, Fong EJ. Evaluation of crush protection devices for agricultural All-Terrain vehicles. Biosystems Engineering 2019;185:161-73.

18 Lagerstrom E, Hibiske S, Gilkey D, et al. A case study: the development of safety tip sheets for ATV use in Ranching. Safety 2015;1:84-93.

19 Mcintosh AS, Patton DA, Rechnitzer G, et al. Injury mechanisms in fatal Australian quad bike incidents. Traffic Inj Prev 2016;17:386-90.

20 Whiting P, Savović J, Higgins JPT, et al. ROBIS: a new tool to assess risk of bias in systematic reviews was developed. J Clin Epidemiol 2016;69:225-34.

21 Pace R, Pluye P, Bartlett G, et al. Testing the reliability and efficiency of the pilot mixed methods appraisal tool (MMAT) for systematic mixed studies review. Int J Nurs Stud 2012;49:47-53.

22 Haddon W. Advances in the epidemiology of injuries as a basis for public policy. Public Health Rep 1980;95:411-21.

23 Haddon $\mathrm{W}$. The changing approach to the epidemiology, prevention, and amelioration of trauma: the transition to approaches etiologically rather than descriptively based. 1968. Inj Prev 1999;5:231-5.

24 Rustagi N, Kumar A, Norbu L, et al. Applying Haddon matrix for evaluation of road crash victims in Delhi, India. Indian J Surg 2018;80:479-87.

25 Wager E, Wiffen PJ. Ethical issues in preparing and publishing systematic reviews. J Evid Based Med 2011;4:130-4.

26 Cabells Journalytics. Journals under review for Cabells predatory report: Cabells scholarly analytics. Available: https://www2-cabellscom.uaeu.idm.oclc.org/predatory

27 Buergelt PT, Paton D. An ecological risk management and capacity building model. Hum Ecol 2014;42:591-603.

28 Gielen AC, Sleet D. Application of behavior-change theories and methods to injury prevention. Epidemiol Rev 2003;25:65-76.

29 Shea BJ, Grimshaw JM, Wells GA, et al. Development of AMSTAR: a measurement tool to assess the methodological quality of systematic reviews. BMC Med Res Methodol 2007;7:7-14. 